\title{
Independence of the spin current from the Néel vector orientation in antiferromagnet $\mathrm{CoO}$
}

\author{
M. Yang, ${ }^{1, *}$ Q. Li, ${ }^{1, *},{ }^{*}$ D. Hou $\odot,{ }^{2}$ P. Shafer, ${ }^{3}$ A. T. N’Diaye, ${ }^{3}$ C. Klewe,${ }^{3}$ T. Y. Wang, ${ }^{1}$ Xixiang Zhang, ${ }^{4}$ \\ C. Hwang $\mathbb{0}^{5}$ and Z. Q. Qiu ${ }^{1}{ }^{1, 末}$ \\ ${ }^{1}$ Department of Physics, University of California at Berkeley, Berkeley, California 94720, USA \\ ${ }^{2}$ International Centre for Quantum Design of Functional Materials (ICQD), Hefei National Laboratory for Physical Sciences \\ at the Microscale (HFNL), and Synergetic Innovation Center of Quantum Information and Quantum Physics, \\ University of Science and Technology of China, Hefei 230026, China \\ ${ }^{3}$ Advanced Light Source, Lawrence Berkeley National Laboratory, Berkeley, California 94720, USA \\ ${ }^{4}$ Physical Science and Engineering Division (PSE), King Abdullah University of Science and Technology (KAUST), \\ Thuwal 23955-6900, Saudi Arabia \\ ${ }^{5}$ Korea Research Institute of Standards and Science, Yuseong, Daejeon 305-340, Korea
}

(Received 30 March 2020; accepted 29 May 2020; published 11 June 2020)

\begin{abstract}
Spin pumping from ferromagnetic $\mathrm{Fe}$ into antiferromagnetic $\mathrm{CoO}$ across a $\mathrm{Ag}$ spacer layer was studied using ferromagnetic resonance (FMR) in $\mathrm{Py} / \mathrm{CoO} / \mathrm{Ag} / \mathrm{Fe} / \mathrm{Ag}(001)$. The thin Py film on top of $\mathrm{CoO}$ permits an alignment of the CoO Néel vector through field cooling in two otherwise equivalent [110] and [1 10$]$ crystalline axes which are parallel and perpendicular to the Fe magnetization direction, respectively. Fe FMR linewidth is measured as a function of Ag thickness in 10-20-GHz frequency range and in 180-330 K temperature range. We find that there exists an anisotropy in the Fe FMR damping for parallel and perpendicular alignment of the $\mathrm{Fe}$ and $\mathrm{CoO}$ spins. However, such anisotropic damping exists only at thin Ag thickness where there exists a magnetic interlayer coupling between $\mathrm{Fe}$ and $\mathrm{CoO}$, and vanishes at thick $\mathrm{Ag}$ thickness where the interlayer coupling becomes negligible but permitting spin-current transmission into $\mathrm{CoO}$. Our result indicates the absence of anisotropic spin current for parallel and perpendicular alignment of the $\mathrm{Fe}$ and $\mathrm{CoO}$ spin axes.
\end{abstract}

DOI: 10.1103/PhysRevB.101.224418

\section{INTRODUCTION}

Research on spin current across antiferromagnetic (AFM) insulators has attracted much interest after the demonstration of $\mathrm{AFM} \mathrm{NiO}$ and $\mathrm{CoO}$ as excellent spin-current mediators [1-4]. It is generally believed that thermally excited AFM spin modes (magnons) are responsible for the spin-current transmission in an AFM insulator [5-8], especially in the vicinity of the Néel temperature $\left(T_{\mathrm{N}}\right)$ associated with AFM order. An important consequence of the above mechanism is that only the component of spin that is parallel to the AFM Néel vector should be transmitted. This is because AFM magnons oscillate at high frequency ( $\mathrm{THz}$ range) compared to typical current pulses (DC to $\mathrm{GHz}$ ) and their time-averaged spin is effectively parallel to the Néel vector [9-11]. In other words, pumping spin into an AFM should exhibit a strong anisotropic dependence on the AFM spin orientation.

It is obvious that whether or not there exists a spin current anisotropy is crucial to the understanding of spin current in AFM. Experimental verification of this anisotropic behavior of the spin current in AFM, however, seems to show inconclusive results with some reports supporting anisotropic behavior [12-17] and others that are contradictory [18-20]. The complexity in identifying the spin-current anisotropy can be traced to two critical experimental issues. First, many studies-

\footnotetext{
*These authors contributed equally to this work.

†qianli2015@berkeley.edu

‡qiu@berkeley.edu
}

especially those which employed polycrystalline AFM-have assumed a parallel alignment between the ferromagnetic (FM) magnetization and the AFM Néel vector. This assumption is generally not supported by experimental evidence. In fact, many FM/AFM systems are shown to have perpendicular alignment between the FM magnetization and the AFM Néel vector [21-23]. Second, there often exist other effects which coexist with the spin-current effect in the experimental measurement. For example, it was shown that measurements of the inverse spin Hall effect are also susceptible to detecting the proximity effect [24], and the interface morphology could play a role in spin Hall magnetoresistance [25], so that it is sometimes difficult to identify the spin-current contribution quantitatively. The anisotropic linewidth broadening of ferromagnetic resonance (FMR), which is usually used in spinpumping measurements, could also arise in the FM layer from anisotropic spin relaxation, such as two-magnon scattering [26-29], and anisotropic Gilbert damping [30,31], etc. In order to prove/disprove the spin-current anisotropic effect unambiguously, which is crucial to the understanding of spin current in AFM, it is important to have a well-defined system in which the AFM Néel vector orientation can be determined directly by experiment, and to carefully account for other experimental contributions to rule out any other artifacts in the spin-current measurement.

\section{EXPERIMENTAL}

In this paper, we report our investigation of spin current pumped into AFM $\mathrm{CoO}$ from Fe (driven into FMR) across 
(a)

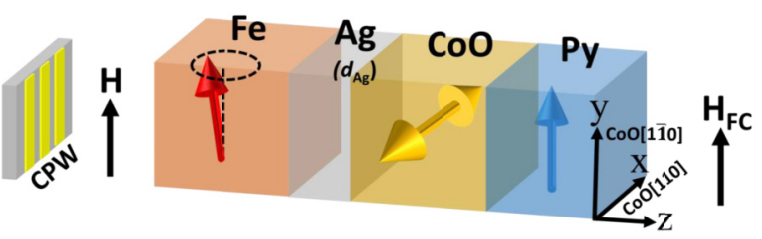

(b)

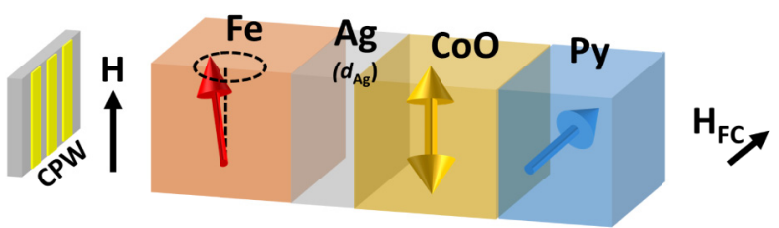

(c)

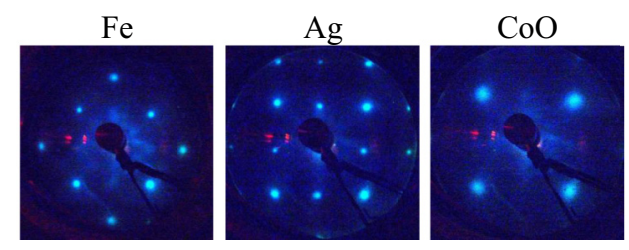

FIG. 1. (a), (b) Schematic drawing of the sample structure and FMR measurement. Here $x$ - and $y$ axes are defined as CoO [110] and [1 $1 \overline{1} 0]$ axes, and $z$ axis is the sample normal direction. In the FMR measurement, the external field $(H)$ is applied along the CPW strip line direction ( $y$ axis). Thus field cooling $\left(H_{\mathrm{FC}}\right)$ and $\mathrm{Py} / \mathrm{CoO}$ coupling create (a) perpendicular and (b) parallel alignment between the frozen $\mathrm{CoO}$ spin Néel vector (yellow arrows) and the Fe magnetization (red arrows). Blue arrows indicate the Py magnetization during field cooling. (c) LEED patterns from sample of $\mathrm{MgO}(3 \mathrm{~nm}) /$ $\mathrm{Py}(2 \mathrm{~nm}) / \mathrm{CoO}(10 \mathrm{~nm}) / \mathrm{Ag}(8 \mathrm{~nm}) / \mathrm{Fe}(4 \mathrm{~nm}) / \mathrm{Ag}(001)$.

a $\mathrm{Ag}$ spacer in a layered structure of $\mathrm{MgO}(3 \mathrm{~nm}) / \mathrm{Py}(2 \mathrm{~nm}) /$ $\mathrm{CoO}(10 \mathrm{~nm}) / \mathrm{Ag}\left(d_{\mathrm{Ag}}\right) / \mathrm{Fe}(4 \mathrm{~nm}) / \mathrm{Ag}(001) \quad$ [Figs. 1(a) and 1(b)], where Py indicates $\mathrm{Ni}_{80} \mathrm{Fe}_{20}$ (permalloy). FMR has been a powerful mechanism for the study of spin pumping because the FM layer at FMR is a high-quality source of spin current that simultaneously serves as a spin-pumping indicator due to FMR linewidth broadening or damping enhancement $[12,16,20,32-36]$. In order to avoid experimental artifacts mentioned previously, we designed our sample by considering the following factors. First, the $\mathrm{CoO}$ AFM spin axis $\left(\stackrel{\leftrightarrow}{S}_{\mathrm{CoO}}\right)$ was controlled by the direction of an applied field $\left(H_{\mathrm{FC}}\right)$ when cooling below $T_{\mathrm{N}}$ with the aid of $\mathrm{Py} / \mathrm{CoO}$ interfacial coupling. In this way $\stackrel{\leftrightarrow}{S}_{\mathrm{CoO}}$ was deterministically aligned along one of two equivalent $\mathrm{CoO}$ easy axes (EA) in the film plane, [110] or [11̄0], "frozen" against subsequent field perturbation [22,37]. Consequently, perpendicular and parallel alignments between the Fe magnetization $\left(\vec{M}_{\mathrm{Fe}}\right)$ and the CoO Néel vector orientation were realized, with the Fe magnetization aligned to the $y$ axis by FMR field $(H)$ during FMR measurement [Figs. 1(a) and 1(b)]. Since both $\mathrm{Fe}$ and $\mathrm{CoO}$ spins in these two situations are always along equivalent crystal axes within each layer, any crystalline anisotropic spin relaxation effect can be ruled out in our experiment. Second, we measured the CoO Néel vector orientation directly by $\mathrm{x}$-ray magnetic linear dichroism (XMLD) spectroscopy to avoid any ambiguity on the relative orientation between the Fe magnetization and the $\mathrm{CoO}$ Néel vector. Finally, the $\mathrm{CoO} / \mathrm{Ag} / \mathrm{Fe}$ interlayer coupling is tuned by the Ag spacer layer thickness to facilitate identification of the interlayer coupling and spin-current contributions to FMR linewidth broadening measurements. Noting that the Ag thickness range $\left(d_{\mathrm{Ag}} \leqslant 8 \mathrm{~nm}\right)$ in our experiment is well below the spin-current diffusion length in $\mathrm{Ag}(\sim 170 \mathrm{~nm})$ [38], we proceeded to carefully search for the spin-current anisotropy effect by comparing the Fe FMR linewidths for parallel $\left(\vec{M}_{\mathrm{Fe}} \| \stackrel{\leftrightarrow}{S}_{\mathrm{CoO}}\right)$ and perpendicular $\left(\vec{M}_{\mathrm{Fe}} \perp \overleftrightarrow{S}_{\mathrm{CoO}}\right)$ alignments between $\vec{M}_{\mathrm{Fe}}$ and $\stackrel{\leftrightarrow}{S}_{\mathrm{CoO}}$.

$\mathrm{MgO} / \mathrm{Py} / \mathrm{CoO} / \mathrm{Ag}\left(d_{\mathrm{Ag}}\right) / \mathrm{Fe} / \mathrm{Ag}(001) \quad$ multilayer samples were prepared in an ultrahigh vacuum system by molecularbeam epitaxy (MBE). The $\operatorname{Ag}(001)$ substrates were prepared by cycles of $\mathrm{Ar}^{+}$ion sputtering at $2 \mathrm{keV}$ and annealing at $500{ }^{\circ} \mathrm{C}$. A 4-nm Fe film was deposited onto the $\operatorname{Ag}(001)$ substrate. Then, a Ag film was deposited with thickness of $2,2.5,3,4.5,6$, and $8 \mathrm{~nm}$ for six samples, respectively. A $10-\mathrm{nm}-\mathrm{CoO}$ film was grown by evaporating $\mathrm{Co}$ at an oxygen atmosphere of $2.0 \times 10^{-6}$ Torr. Then half of the sample surface was coated by a $2-\mathrm{nm}$ Py film. Finally, a $3-\mathrm{nm} \mathrm{MgO}$ capping layer was deposited on the entire sample for protection of the metallic layers against ambient oxidation. All films were grown at room temperature. In the sample structure, the $\mathrm{Fe}$, $\mathrm{Ag}$, and $\mathrm{CoO}$ layers are epitaxially grown with the in-plane lattice relation of $\mathrm{CoO}[110]\|\mathrm{Ag}[110]\| \mathrm{Fe}[100] \| \mathrm{Ag}[110]$ [see the low-energy electron diffraction (LEED) patterns in Fig. 1(c)]. FMR measurements were performed by placing the sample on top of a coplanar waveguide (CPW) in the temperature range of 180 to $320 \mathrm{~K}$. FMR signal was detected by collecting the field derivative of the absorption intensity. FMR signal of 4-nm Fe layer and 2-nm Py layer can be distinguished by different resonance field [39] and resonance intensity [40]. In fact, the Py FMR signal below the $\mathrm{CoO}$ Néel temperature is virtually invisible in our system. Hysteresis loops of the Fe and Py layers were obtained by magneto-optical Kerr effect (MOKE) measurements on the half of sample without Py layer and X-ray magnetic circular dichroism (XMCD) spectroscopy with x-ray photon energy tuned at Ni absorption peak, respectively. XMCD and XMLD measurements were performed at beamlines 4.0.2 and 6.3.1 of the Advanced Light Source.

\section{RESULTS AND DISCUSSIONS}

We first studied the interlayer coupling between $\mathrm{Fe}$ and $\mathrm{CoO}$ across the $\mathrm{Ag}$ spacer layer in $\mathrm{CoO} / \mathrm{Ag} / \mathrm{Fe} / \mathrm{Ag}(001)$ by measuring the $\mathrm{Fe}$ hysteresis loops after field cooling [Figs. 2(a) and 2(b)]. Square easy-axis hysteresis loops were obtained for $H \| H_{\mathrm{FC}}$ and double-split hard-axis (HA) loops were obtained for $H \perp H_{\mathrm{FC}}$, indicating that the $\mathrm{Fe} / \mathrm{CoO}$ interlayer coupling has induced an in-plane uniaxial anisotropy in the Fe film with the EA parallel to $H_{\mathrm{FC}}$ which is consistent with literature results [41]. Both the coercivity $\left(H_{\mathrm{C}}\right)$ and the shifted field $\left(H_{\mathrm{S}}\right.$, defined in Fig. $2(\mathrm{~b})$, which is proportional to the uniaxial anisotropy [42]) decrease with increasing $\mathrm{Ag}$ thickness and approach constant values for $d_{\mathrm{Ag}}$ above $4.5 \mathrm{~nm}$ [43] [see the summary result shown later in Fig. 3(e)]. It should be mentioned that the interlayer coupling between $\mathrm{Fe}$ and $\mathrm{CoO}$ layers across a thin $\mathrm{Ag}$ layer could even exhibit weak oscillatory behavior for very high-quality films [43], but the 

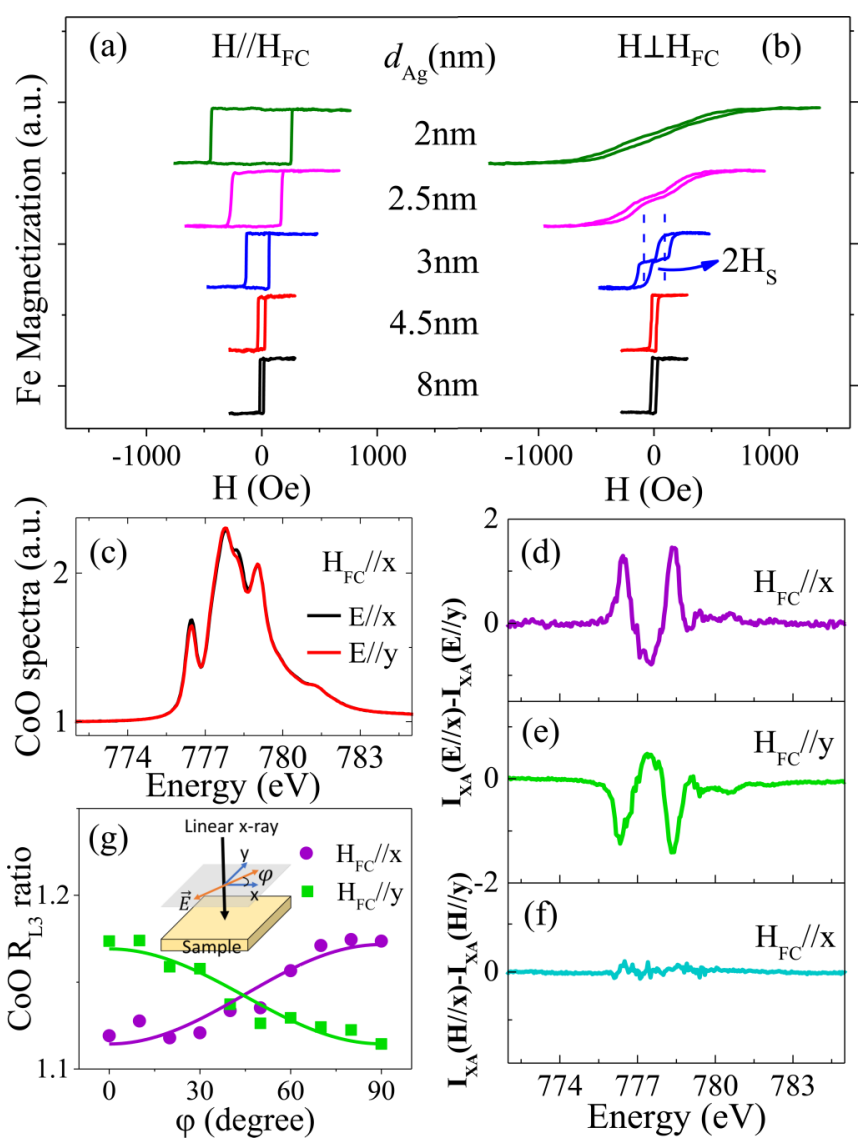

FIG. 2. Fe hysteresis loops for (a) $H \| H_{\mathrm{FC}}$ and (b) $H \perp H_{\mathrm{FC}}$. The interlayer coupling between $\mathrm{Fe}$ and $\mathrm{CoO}$ decreases with increasing $\mathrm{Ag}$ thickness and vanishes above 4.5-nm Ag thickness. (c) CoO spectra measured with linear polarized $\mathrm{x}$ rays for $H_{\mathrm{FC}} \| x$. XMLD spectra of (d) $H_{\mathrm{FC}} \| x$ and (e) $H_{\mathrm{FC}} \| y$ are equal and opposite, confirming that $\mathrm{CoO}$ spins are aligned along $y$ and $x$ directions, respectively. (f) The unchanged XMLD (with fixed x-ray polarization, $E \| x$ and $E \| y$ ) after rotating a 4000-Oe field from $x$ to $y$ axis proves the frozen $\mathrm{CoO}$ spins after field cooling. (g) The $\mathrm{CoO} R_{\mathrm{L} 3}$ ratio exhibits $\cos ^{2} \varphi$ dependence on $\mathrm{x}$-ray polarization angle $(\varphi)$, showing that $\mathrm{CoO}$ spins are aligned perpendicularly to $H_{\mathrm{FC}}$. Solid lines are cosine fits to the data. Inset shows the schematic of XMLD measurement, with $\varphi$ defined as the angle between x-ray polarization and $x$ axis. All measurements were performed at $200 \mathrm{~K}$.

coupling usually decreases monotonically with spacer layer thickness when films become rougher than epitaxial metallic thin films (e.g., $\mathrm{CoO}$ film grown by MBE). Nevertheless the $\mathrm{Fe} / \mathrm{CoO}$ interlayer coupling vanishes above 4.5-nm Ag thickness, which is important for the FMR linewidth study presented later.

We then performed XMLD spectroscopy at $\operatorname{Co} L_{3}$ edge to determine the $\mathrm{CoO}$ AFM spin orientation in $\mathrm{MgO} / \mathrm{Py} / \mathrm{CoO} / \mathrm{Ag} / \mathrm{Fe} / \mathrm{Ag}(001)$. Because FMR is performed by applying the magnetic field along the CPW stripe line direction [H $\| y$ in both Figs. 1(a) and 1(b)], we controlled the CoO spin orientation by field cooling while leveraging $\mathrm{Py} / \mathrm{CoO}$ coupling under the conditions of $H_{\mathrm{FC}} \| y$ [Fig. 1(a)] and $H_{\mathrm{FC}} \| x$ [Fig. 1(b)], respectively. The CoO spectra measured with two orthogonal x-ray polarizations [44] [Fig. 2(c)] were used to obtain x-ray linear dichroism [Figs. 2(d) and 2(e)]
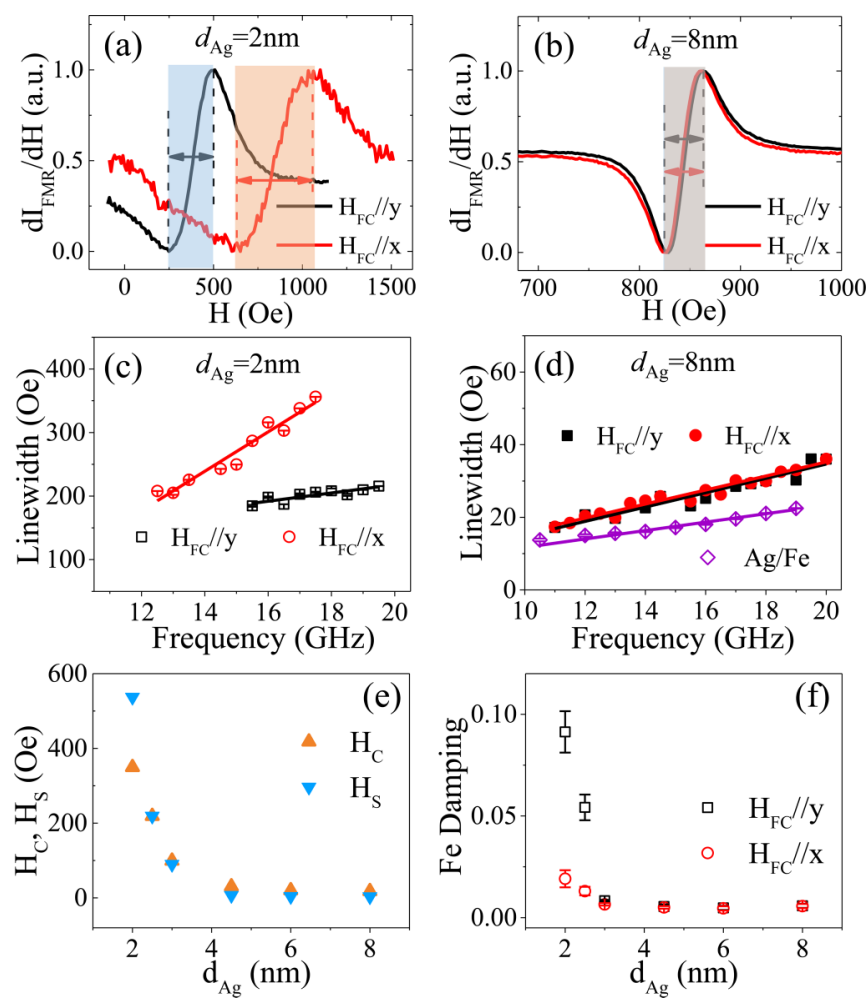

FIG. 3. Fe FMR spectra measured at $17.5 \mathrm{GHz}$ with $H_{\mathrm{FC}} \| x$ and $H_{\mathrm{FC}} \| y$ in (a) $d_{\mathrm{Ag}}=2$-nm sample and (b) $d_{\mathrm{Ag}}=8$-nm sample. The shading with arrows indicates the FMR full linewidth $(2 \Delta H)$. Frequency-dependent FMR linewidth $(\Delta H)$ for $H_{\mathrm{FC}} \| y$ and $H_{\mathrm{FC}} \| x$ from (c) $d_{\mathrm{Ag}}=2$-nm sample and (d) $d_{\mathrm{Ag}}=8$-nm sample. Solid lines are fitting with Eq. (1). The linewidth from a reference sample of $\mathrm{Ag}(8 \mathrm{~nm}) / \mathrm{Fe}(4 \mathrm{~nm})$ is also shown in (d) to indicate the existence of spin-current pumping into $\mathrm{CoO}$. (e) Coercivity $\left(H_{\mathrm{C}}\right)$, splitting field $\left(H_{\mathrm{S}}\right)$, and (f) FMR damping of Fe layer as a function of the $\mathrm{Ag}$ spacer layer thickness. All measurements were performed at $200 \mathrm{~K}$.

for two field-cooling directions. The inverse intensity of these dichroism spectra and their comparison to previous studies $[22,44]$ indicate that the $\mathrm{CoO}$ spin axis is along the $y$ axis $\left(\stackrel{\leftrightarrow}{S}_{\mathrm{CoO}} \| y\right)$ for $H_{\mathrm{FC}} \| x$ and along the $x$ axis $\left(\stackrel{\leftrightarrow}{S}_{\mathrm{CoO}} \| x\right)$ for $H_{\mathrm{FC}} \| y$, proving that the $\mathrm{Py} / \mathrm{CoO}$ coupling leads to a perpendicular alignment between the Py magnetization and the $\mathrm{CoO}$ spin axis $\left(\vec{M}_{\mathrm{Py}} \perp \stackrel{\leftrightarrow}{S}_{\mathrm{CoO}}\right)$, which is a feature of spin-flop coupling. This conclusion is further confirmed by the observed $\cos ^{2} \varphi$ (here $\varphi$ is the X-ray polarization angle) dependence of the $\mathrm{CoO} R_{\mathrm{L} 3}$ ratio [Fig. $2(\mathrm{~g})$ ], which is defined as intensity ratio of the peak at $777.8 \mathrm{eV}$ over the peak at $778.2 \mathrm{eV}$. Then for the case of $H_{\mathrm{FC}} \| y$ [Fig. 1(a)], the Fe FMR is under the condition of $\vec{M}_{\mathrm{Fe}} \perp \stackrel{\leftrightarrow}{S}_{\mathrm{CoO}}$. For the case of $H_{\mathrm{FC}} \| x$ [Fig 1(b)], it is important to additionally verify that the $\mathrm{CoO}$ spins stay frozen along the $y$ axis after the external FMR field rotates the Fe and Py magnetization from $x$ - to $y$ axis. Figure 2(f) shows that the CoO XMLD signal remains unchanged after switching the Fe magnetization from $x$ - to $y$ axis by a 4000-Oe magnetic field, proving that $\mathrm{CoO}$ spins remain frozen [22] along the $\mathrm{y}$ axis after field cooling $\left(H_{\mathrm{FC}} \| x\right)$. Thus for the case of $H_{\mathrm{FC}} \| x$ [Fig. 1(b)], we ensure that the Fe FMR is performed under the condition of $\vec{M}_{\mathrm{Fe}} \| \stackrel{\leftrightarrow}{S}_{\mathrm{CoO}}$. 
Next we performed Fe FMR measurements at $200 \mathrm{~K}$ by applying an external magnetic field $(H)$ along the CPW strip line direction $(y$ axis) to investigate spin pumping from the Fe layer for the two situations of $\vec{M}_{\mathrm{Fe}} \perp \overleftrightarrow{S}_{\mathrm{CoO}}$ [Fig. 1(a)] and $\vec{M}_{\mathrm{Fe}} \| \overleftrightarrow{S}_{\mathrm{CoO}}$ [Fig. 1(b)], respectively. We find that the Fe FMR in the $d_{\mathrm{Ag}}=2$-nm sample produces substantially different spectra of the FMR differential intensity $\left(d I_{\mathrm{FMR}} / d H\right)$ for $\vec{M}_{\mathrm{Fe}} \perp \stackrel{\leftrightarrow}{S}_{\mathrm{CoO}}$ and $\vec{M}_{\mathrm{Fe}} \| \overleftrightarrow{S}_{\mathrm{CoO}}$ [Fig. 3(a)]. First, the FMR resonance field is clearly shifted to a higher field for $\vec{M}_{\mathrm{Fe}} \| \overleftrightarrow{S}_{\mathrm{CoO}}$ than for $\vec{M}_{\mathrm{Fe}} \perp \overleftrightarrow{S}_{\mathrm{CoO}}$, which is consistent with the induced uniaxial anisotropy by the $\mathrm{Fe} / \mathrm{CoO}$ perpendicular interlayer coupling across the Ag spacer layer [Fig. 2(b)]. Second, the FMR linewidth $2 \Delta H$ ( $\Delta H$ is defined as half width at half maximum from the Lorentz derivative fit) shows an obvious broadening for $\vec{M}_{\mathrm{Fe}} \| \overleftrightarrow{S}_{\mathrm{CoO}}$ compared to $\vec{M}_{\mathrm{Fe}} \perp \overleftrightarrow{S}_{\mathrm{CoO}}$. Since FMR linewidth at a single frequency does not necessarily reflect the intrinsic damping, we performed FMR in the frequency range of $10-20 \mathrm{GHz}$ [Fig. 3(c)] to obtain the frequency-dependent FMR linewidth $\Delta H$. It is observed that $\Delta H$ follows the expected linear dependence on frequency $(f)$ with the slope $\alpha$ representing the intrinsic FMR damping [45].

$$
\Delta H=\Delta H_{0}+\frac{2 \pi}{\mu_{0} \gamma} \alpha f .
$$

Here $\Delta H_{0}$ is intercept of linewidth at zero frequency, and $\gamma$ is gyromagnetic ratio with $\gamma / 2 \pi \approx 29.4 \mathrm{GHz} / \mathrm{T}$. A linear fitting (solid lines) of Fig. 3(c) using Eq. (1) yields $\alpha=$ 0.091 for the case of $\vec{M}_{\mathrm{Fe}} \| \stackrel{\leftrightarrow}{S}_{\mathrm{CoO}}$ and $\alpha=0.019$ for the case

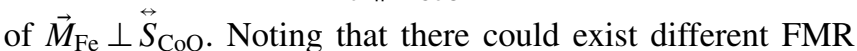
damping for $H \| y$ and $H \|-y$ due to exchange bias for the case of $H_{\mathrm{FC}} \| y$, we also measured the FMR linewidth for $H \|-y$ and find the same intrinsic damping as that for $H \| y$. Recalling that the $\mathrm{CoO}$ spins are along equivalent crystal axes for the two cases of $\vec{M}_{\mathrm{Fe}} \| \overleftrightarrow{S}_{\mathrm{CoO}}$ and $\vec{M}_{\mathrm{Fe}} \perp \overleftrightarrow{S}_{\mathrm{CoO}}$, we conclude that the different damping coefficients in the $d_{\mathrm{Ag}}=$ 2-nm sample must be due to change in orientation between $\vec{M}_{\mathrm{Fe}}$ and $\overleftrightarrow{S}_{\mathrm{CoO}}$. Because the spin-current diffusion length in $\mathrm{CoO}(\sim 6 \mathrm{~nm}$ [3]) is shorter than the $10 \mathrm{~nm}$ of $\mathrm{CoO}$ in our sample, the Py layer should not be relevant to spin-current absorption here. Although the FMR damping being greater when $\vec{M}_{\mathrm{Fe}} \| \overleftrightarrow{S}_{\mathrm{CoO}}$ than for $\vec{M}_{\mathrm{Fe}} \perp \overleftrightarrow{S}_{\mathrm{CoO}}$ is consistent with the spin-current anisotropy in AFM, one has to be careful when considering the FM/AFM coupling which could also result in an anisotropic FMR damping via other mechanisms [26-29].

To separate the $\mathrm{Fe} / \mathrm{CoO}$ interlayer coupling effect from the spin-current anisotropy, we performed Fe FMR measurements on the $d_{\mathrm{Ag}}=8-\mathrm{nm}$ sample where the 8-nm Ag is thick enough to diminish the $\mathrm{Fe} / \mathrm{CoO}$ interlayer coupling but thin enough to permit spin-current transmission across the Ag. First, we find that the FMR field in the $d_{\mathrm{Ag}}=8-\mathrm{nm}$ sample is identical for the cases of $\vec{M}_{\mathrm{Fe}} \| \overleftrightarrow{S}_{\mathrm{CoO}}$ and $\vec{M}_{\mathrm{Fe}} \perp \overleftrightarrow{S}_{\mathrm{CoO}}$ [Fig. 3(b)], confirming the vanished $\mathrm{Fe} / \mathrm{CoO}$ interlayer coupling across 8-nm Ag, which agrees with the isotropy of the corresponding hysteresis loops in Figs. 2(a) and 2(b). Second, the FMR damping $(\alpha=0.0057)$ is greater than that from a $\mathrm{Ag} / \mathrm{Fe} / \mathrm{Ag}(001)$ reference film ( $\alpha=0.0034)$ [Fig. 3(d)], showing that 8-nm Ag
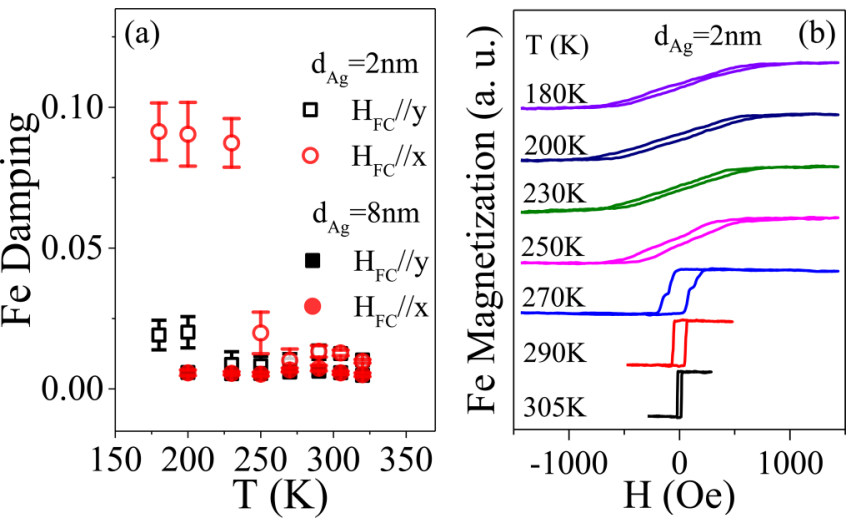

FIG. 4. (a) Fe FMR damping for $H_{\mathrm{FC}} \| x$ and $H_{\mathrm{FC}} \| y$ as a function of temperature from $d_{\mathrm{Ag}}=2$ - and $d_{\mathrm{Ag}}=8$-nm samples. (b) Fe hysteresis loops of $d_{\mathrm{Ag}}=2 \mathrm{~nm}$ sample for $H \perp H_{\mathrm{FC}}$.

indeed is thin enough to permit spin-current transmission into the $\mathrm{CoO}$ layer. Finally, the identical damping $(\alpha=0.0057)$ within the accuracy of 0.0007 for the cases of $\vec{M}_{\mathrm{Fe}} \| \overleftrightarrow{S}_{\mathrm{CoO}}$ and $\vec{M}_{\mathrm{Fe}} \perp \stackrel{\leftrightarrow}{\mathrm{CoO}}_{\mathrm{C}}$ [Fig. 3(d)] shows the absence of anisotropic FMR damping. By comparing the FMR results from the $d_{\mathrm{Ag}}=$ 2-nm and $d_{\mathrm{Ag}}=8-\mathrm{nm}$ samples, the anisotropic damping of Fe FMR in the $d_{\mathrm{Ag}}=2 \mathrm{~nm}$ sample must come entirely from the $\mathrm{Fe} / \mathrm{CoO}$ interlayer coupling effect rather than from any spin-current anisotropy. To further confirm this assertion, we measured the FMR damping coefficients systematically for the cases of $\vec{M}_{\mathrm{Fe}} \| \stackrel{\leftrightarrow}{S}_{\mathrm{CoO}}$ and $\vec{M}_{\mathrm{Fe}} \perp \overleftrightarrow{S}_{\mathrm{CoO}}$ as a function of $\mathrm{Ag}$ thickness. The result [Fig. 3(f)] shows that the damping coefficients for $\vec{M}_{\mathrm{Fe}} \| \stackrel{\leftrightarrow}{S}_{\mathrm{CoO}}$ and $\vec{M}_{\mathrm{Fe}} \perp \stackrel{S}{\mathrm{CoO}}_{\mathrm{Cre}}$ aifferent only for $d_{\mathrm{Ag}}<4.5 \mathrm{~nm}$ where there exists $\mathrm{Fe} / \mathrm{CoO}$ interlayer coupling across the Ag spacer layer as evidenced by the enhanced $H_{\mathrm{C}}$ and $H_{\mathrm{S}}$ in the hysteresis loops [Fig. 3(e)]. Extracted from the FMR result, fourfold anisotropy of the Fe layer is about 496.2 Oe. The interlayer coupling-induced uniaxial anisotropy of Fe layer in $d_{\mathrm{Ag}}=2$-nm sample is about $549.1 \mathrm{Oe}$, which is consistent with the MOKE result [Fig. 2(b)].

To further confirm that the anisotropic damping in samples with the Ag spacer layer below $4.5 \mathrm{~nm}$ is indeed from the $\mathrm{Fe} / \mathrm{CoO}$ interlayer coupling, we performed a temperaturedependent study on the $d_{\mathrm{Ag}}=2$-nm sample. Fe hysteresis loops [Fig. 4(b)] for $H \perp H_{\mathrm{FC}}$ show that the HA loop character (high saturation field and low remanence) evolves into an EA loop character (square-shape loop with a full remanence) above $T \sim 270-290 \mathrm{~K}$ which corresponds to the $\mathrm{CoO}$ Néel temperature [46]. Correspondingly, the Fe FMR damping anisotropy between the two cases of $\vec{M}_{\mathrm{Fe}} \perp \overleftrightarrow{S}_{\mathrm{CoO}}$ and $\vec{M}_{\mathrm{Fe}} \|$ $\overleftrightarrow{S}_{\text {CoO }}$ is negligibly small above $270 \mathrm{~K}$ and develops rapidly below $270 \mathrm{~K}$ in the $d_{\mathrm{Ag}}=2-\mathrm{nm}$ sample. In contrast, the Fe FMR damping exhibits negligible anisotropy across the entire temperature range for the $d_{\mathrm{Ag}}=8$-nm sample. We also note that the Fe FMR damping coefficients in both $d_{\mathrm{Ag}}=2$ and $d_{\mathrm{Ag}}=8$-nm samples seem to show a broad peak in the temperature range of $\sim 270-300 \mathrm{~K}$. Because these damping values are approaching the limits of accuracy for the FMR measurements, we hesitate to claim this peak as significant. However, it is worth mentioning that an enhancement of 
spin-current injection into AFM $\mathrm{CoO}$ in the vicinity of $\mathrm{CoO}$ Néel temperature has been reported in the literature and has been attributed to excitation of thermal magnons in $\mathrm{CoO}[3,4,19]$. Based on the damping anisotropy between $\vec{M}_{\mathrm{Fe}} \perp \overleftrightarrow{S}_{\mathrm{CoO}}$ and $\vec{M}_{\mathrm{Fe}} \| \overleftrightarrow{S}_{\mathrm{CoO}}$ in the $d_{\mathrm{Ag}}=2$-nm sample at low temperature, which is much greater than the damping in the $d_{\mathrm{Ag}}=8$-nm sample, we further conclude that this anisotropic damping is entirely from interlayer coupling rather than any spin-current anisotropy. Despite the weak FMR signal, our result is enough to support our main conclusion here. Finally we note that there have been reports on anisotropic damping in FM/AFM systems via other interfacial mechanisms, such as two-magnon scattering [28,29]. The current work is focused on separating the effects of spin-current anisotropy from those of interlayer coupling in terms of their influence on magnetic damping anisotropy, reserving a more detailed study of the exact nature of interfacial coupling to future studies.

\section{CONCLUSIONS}

In summary, we studied the damping of Fe ferromagnetic resonance in $\mathrm{Py} / \mathrm{CoO} / \mathrm{Ag} / \mathrm{Fe}$ multilayer samples. By determining the CoO Néel vector orientation using XMLD spectroscopy and separating the interlayer coupling effect from the spin-current effect, we show that there is no detectable spin-current anisotropy within the accuracy of 0.0007 of the damping values for $\mathrm{CoO}$ spins parallel and perpendicular to the Fe magnetization. All observed anisotropic Fe FMR damping is entirely due to the $\mathrm{Fe} / \mathrm{CoO}$ interlayer coupling across the Ag spacer layer. Our result on the absence of spincurrent anisotropy relative to the $\mathrm{CoO}$ Néel vector orientation establishes a criterion for future studies on the spin current in antiferromagnetic insulators.

\section{ACKNOWLEDGMENTS}

The project was primarily supported by US Department of Energy (DOE), Office of Science, Office of Basic Energy Sciences, Materials Sciences and Engineering Division under Contract No. DE-AC02-05CH112311 (van der Waals heterostructures program, KCWF16). This work was also supported by King Abdullah University of Science and Technology (KAUST), Office of Sponsored Research (OSR) and under the Award No. OSR-2017-CRG6-3427, Future Materials Discovery Program through the National Research Foundation of Korea (Grant No. 2015M3D1A1070467), Science Research Center Program through the National Research Foundation of Korea (Grant No. 2015R1A5A1009962). This research used resources of the Advanced Light Source, which is a DOE Office of Science User Facility under Contract No. DE-AC02-05CH11231.
[1] H. Wang, C. Du, P. C. Hammel, and F. Yang, Phys. Rev. Lett. 113, 097202 (2014).

[2] C. Hahn, G. de Loubens, V. V. Naletov, J. Ben Youssef, O. Klein, and M. Viret, Europhys. Lett. 108, 57005 (2014).

[3] W. Lin, K. Chen, S. Zhang, and C. L. Chien, Phys. Rev. Lett. 116, 186601 (2016).

[4] Z. Qiu, J. Li, D. Hou, E. Arenholz, A. T. N'Diaye, A. Tan, K. Uchida, K. Sato, S. Okamoto, Y. Tserkovnyak, Z. Q. Qiu, and E. Saitoh, Nat. Commun. 7, 12670 (2016).

[5] K. M. D. Hals, Y. Tserkovnyak, and A. Brataas, Phys. Rev. Lett. 106, 107206 (2011).

[6] M. W Daniels, W. Guo, G. M. Stocks, D. Xiao, and J. Xiao, New J. Phys. 17, 103039 (2015).

[7] S. M. Rezende, R. L. Rodríguez-Suárez, and A. Azevedo, Phys. Rev. B 93, 054412 (2016).

[8] K. Chen, W. Lin, C. L. Chien, and S. Zhang, Phys. Rev. B 94, 054413 (2016).

[9] S. Takei, T. Moriyama, T. Ono, and Y. Tserkovnyak, Phys. Rev. B 92, 020409(R) (2015).

[10] T. Kampfrath, A. Sell, G. Klatt, A. Pashkin, S. Mährlein, T. Dekorsy, M. Wolf, M. Fiebig, A. Leitenstorfer, and R. Huber, Nat. Photon. 5, 31 (2011).

[11] R. Cheng, J. Xiao, Q. Niu, and A. Brataas, Phys. Rev. Lett. 113, 057601 (2014).

[12] T. Moriyama, M. Kamiya, K. Oda, K. Tanaka, K.-J. Kim, and T. Ono, Phys. Rev. Lett. 119, 267204 (2017).

[13] A. A. Baker, A. I. Figueroa, C. J. Love, S. A. Cavill, T. Hesjedal, and G. van der Laan, Phys. Rev. Lett. 116, 047201 (2016).
[14] R. Lebrun, A. Ross, S. A. Bender, A. Qaiumzadeh, L. Baldrati, J. Cramer, A. Brataas, R. A. Duine, and M. Kläui, Nature (London) 561, 222 (2018).

[15] Z. Qiu, D. Hou, J. Barker, K. Yamamoto, O. Gomonay, and E. Saitoh, Nat. Mater. 17, 577 (2018).

[16] D. M. Polishchuk, A. Kamra, T. I. Polek, A. Brataas, and V. Korenivski, Phys. Rev. Lett. 123, 247201 (2019).

[17] D. Hou, Z. Qiu, J. Barker, K. Sato, K. Yamamoto, S. Vélez, J. M. Gomez-Perez, L. E. Hueso, F. Casanova, and E. Saitoh, Phys. Rev. Lett. 118, 147202 (2017).

[18] H. Saglam, J. C. Rojas-Sanchez, S. Petit, M. Hehn, W. Zhang, J. E. Pearson, S. Mangin, and A. Hoffmann, Phys. Rev. B 98, 094407 (2018).

[19] Q. Li, M. Yang, C. Klewe, P. Shafer, A. T. N'Diaye, D. Hou, T. Y. Wang, N. Gao, E. Saitoh, C. Hwang, R. J. Hicken, J. Li, E. Arenholz, and Z. Q. Qiu, Nat. Commun. 10, 5265 (2019).

[20] B. Khodadadi, Y. Lim, D. A. Smith, R. W. Greening, Y. Zheng, Z. Diao, C. Kaiser, and S. Emori, Phys. Rev. B 99, 024435 (2019).

[21] E. Arenholz, G. van der Laan, R. V. Chopdekar, and Y. Suzuki, Phys. Rev. Lett. 98, 197201 (2007).

[22] J. Wu, J. S. Park, W. Kim, E. Arenholz, M. Liberati, A. Scholl, Y. Z. Wu, C. Hwang, and Z. Q. Qiu, Phys. Rev. Lett. 104, 217204 (2010).

[23] Y. Takamura, E. Folven, J. B. R. Shu, K. R. Lukes, B. Li, A. Scholl, A. T. Young, S. T. Retterer, T. Tybell, and J. K. Grepstad, Phys. Rev. Lett. 111, 107201 (2013). 
[24] Y. M. Lu, Y. Choi, C. M. Ortega, X. M. Cheng, J. W. Cai, S. Y. Huang, L. Sun, and C. L. Chien, Phys. Rev. Lett. 110, 147207 (2013).

[25] T. K. H. Pham, M. Ribeiro, J. H. Park, N. J. Lee, K. H. Kang, E. Park, V. Q. Nguyen, A. Michel, C. S. Yoon, S. Cho, and T. H. Kim, Sci. Rep. 8, 13907 (2018).

[26] R. D. McMichael, M. D. Stiles, P. J. Chen, and W. F. Egelhoff, J. Appl. Phys. 83, 7037 (1998).

[27] M. J. Pechan, D. Bennett, N. Teng, C. Leighton, J. Nogués, and I. K. Schuller, Phys. Rev. B 65, 064410 (2002).

[28] T. Mewes, R. L. Stamps, H. Lee, E. Edwards, M. Bradford, C. K. A. Mewes, Z. Tadisina, and S. Gupta, IEEE Magn. Lett. 1, 3500204 (2010).

[29] J. Beik Mohammadi, J. M. Jones, S. Paul, B. Khodadadi, C. K. A. Mewes, T. Mewes, and C. Kaiser, Phys. Rev. B 95, 064414 (2017).

[30] L. Chen, S. Mankovsky, S. Wimmer, M. A. W. Schoen, H. S. Körner, M. Kronseder, D. Schuh, D. Bougeard, H. Ebert, D. Weiss, and C. H. Back, Nat. Phys. 14, 490 (2018).

[31] Y. Li, F. Zeng, S. S.-L. Zhang, H. Shin, H. Saglam, V. Karakas, O. Ozatay, J. E. Pearson, O. G. Heinonen, Y. Wu, A. Hoffmann, and W. Zhang, Phys. Rev. Lett. 122, 117203 (2019).

[32] R. Urban, G. Woltersdorf, and B. Heinrich, Phys. Rev. Lett. 87, 217204 (2001).

[33] B. Heinrich, Y. Tserkovnyak, G. Woltersdorf, A. Brataas, R. Urban, and G. E.W. Bauer, Phys. Rev. Lett. 90, 187601 (2003).

[34] P. Merodio, A. Ghosh, C. Lemonias, E. Gautier, U. Ebels, M. Chshiev, H. Béa, V. Baltz, and W. E. Bailey, Appl. Phys. Lett. 104, 032406 (2014).
[35] W. Zhang, M. B. Jungfleisch, W. Jiang, J. E. Pearson, A. Hoffmann, F. Freimuth, and Y. Mokrousov, Phys. Rev. Lett. 113, 196602 (2014).

[36] L. Frangou, S. Oyarzún, S. Auffret, L. Vila, S. Gambarelli, and V. Baltz, Phys. Rev. Lett. 116, 077203 (2016).

[37] W. N. Cao, J. Li, G. Chen, J. Zhu, C. R. Hu, and Y. Z. Wu, Appl. Phys. Lett. 98, 262506 (2011).

[38] B. Kardasz, O. Mosendz, B. Heinrich, Z. Liu, and M. Freeman, J. Appl. Phys. 103, 07C509 (2008).

[39] R. E. Camley, Z. Celinski, and R. L. Stamps, in Magnetism of Surfaces, Interfaces, and Nanoscale Materials (Elsevier, Amsterdam, 2015), Vol. 5, Chap. 10, p. 428.

[40] M. H. Seavey and P. E. Tannenwald, J. Appl. Phys. 29, 292 (1958).

[41] Q. Li, G. Chen, T. P. Ma, J. Zhu, A. T. N'Diaye, L. Sun, T. Gu, Y. Huo, J. H. Liang, R. W. Li, C. Won, H. F. Ding, Z. Q. Qiu, and Y. Z. Wu, Phys. Rev. B 91, 134428 (2015).

[42] W. Weber, A. Bischof, R. Allenspach, Ch. Würsch, C. H. Back, and D. Pescia, Phys. Rev. Lett. 76, 3424 (1996).

[43] Y. Meng, J. Li, P.-A. Glans, C. A. Jenkins, E. Arenholz, A. Tan, J. Gibbons, J. S. Park, C. Hwang, H. W. Zhao, and Z. Q. Qiu, Phys. Rev. B 85, 014425 (2012).

[44] G. van der Laan, E. Arenholz, R. V. Chopdekar, and Y. Suzuki, Phys. Rev. B 77, 064407 (2008).

[45] B. Heinrich, J. F. Cochran, and R. Hasegawa, J. Appl. Phys. 57, 3690 (1985).

[46] Q. Li, T. Gu, J. Zhu, Z. Ding, J. X. Li, J. H. Liang, Y. M. Luo, Z. Hu, C. Y. Hua, H.-J. Lin, T. W. Pi, C. Won, and Y. Z. Wu, Phys. Rev. B 91, 104424 (2015). 